\title{
INFORMAÇÕES FUNDAMENTAIS AO GERENCIAMENTO DAS ATIVIDADES DA CADEIA DE VALOR - O CASO DAS PEQUENAS EMPRESAS INDUSTRIAIS DE PRODUTOS ALIMENTARES
}

\author{
Carin Raquel Liczbinski \\ Universidade de Cruz Alta - Departamento de Estudos Econômicos e Administrativos \\ Av. Andrade Neves, 308 - Cruz Alta/RS - CEP 98025-810 \\ Martinho Luís Kelm \\ Universidade Regional do Noroeste do Estado do RS - Departamento de Economia e Contabilidade \\ Rua São Francisco, 501 - Ijuí/RS - CEP 98700-000 \\ Aline França de Abreu \\ Universidade Federal de Santa Catarina - Departamento de Engenharia de Produção e Sistemas \\ Trindade - Caixa Postal 246 - Florianópolis/SC - CEP 88040-900
}

This paper presents a study of the information judged to be the most important in the administration of the activities of the Value Chain in small food-products industries specifically the ones of the betterment, milling, torrefaction, and the make up of vegetal food products, in Rio Grande do Sul, in the perspective of a group of entrepreneurs and experts. The paper emphasizes the subject of the participation of the small businesses in the Brazilian economy is very relevant, what ratifies the importance and attention given to them. Just like the big companies, the small companies can and ought to use the available resources for the best usage and management of them. The information is one of these resources, that when well administered and effectively used helps directly in the decisionmaking process that supports the business, even by the fact of reducing the error chances among the decisions. The formal management of the information is not still a real fact in the great part of the small businesses. However, it may be the basis for definition and consecution of the strategies that aim the competitive advantage.

Keywords - information, value chain, management of the activities.

\section{Introdução}

$\mathrm{O}$ meio no qual as empresas atuam muda permanentemente, obrigando-as a se adaptarem e reagirem aos novos anseios do mercado. Esta é a realidade de grande parte dos negócios atuais, dos mais diversos setores e dos mais diversos portes. Diante disso, a necessidade de melhorar as condições competitivas das empresas é permanentemente crescente. As grandes empresas, normalmente, percebem os sinais de necessidade de melhorias mais cedo que os pequenos negócios. No entanto, todos os empreendimentos devem ter claro que mudanças, melhores práticas de produção e de gestão são necessárias para manter-se no mercado.

As organizações são, também, conjuntos de atividade que devem ser eficiente para que se tenha êxito na empresa como um todo, influenciando seu nível de competitividade. Nesse sentido, o estudo apresenta um método que busca identificar quais as informações fundamentais para a administração das atividades das pequenas indústrias gaúchas, 
considerando-se o caso específico das industriais de produtos alimentares - relacionadas ao ramo de beneficiamento, moagem, torrefação e fabricação de produtos alimentares de origem vegetal.

\section{As Pequenas Empresas Brasileiras}

As pequenas organizações enfrentam diversos obstáculos para se adaptarem ao mercado, em constante mudança, e obter uma posição competitiva favorável. Mesmo assim, conforme destacam Cândido \& Abreu (2000) essas organizações possuem grande presença no cenário mundial e brasileiro.

Considerando a distribuição do número de empresas industriais por faixa de pessoal ocupado, mais de $96 \%$ das empresas industriais brasileiras, sob o critério de número de empregados, eram, em 1994, micro e pequenas empresas (SEBRAE, 2001). Esses números demonstram que a participação da micro e pequena empresa é muito relevante para a economia brasileira e que essas organizações merecem atenção. No entanto, existe outro dado que ofusca estes índices. Uma pesquisa efetuada em 1997 pelo Sebrae em 12 estados brasileiros, mostrou que o índice de falência das empresas varia entre $47 \%$ e $73 \%$ em três anos, de acordo com o estado em que se situam. No mundo inteiro toma-se como base uma estimativa segundo a qual, $80 \%$ das empresas fracassam em três anos de vida (Cohen, 2000, p. 160).

Embora sejam em grande número, há um ciclo constante de nascimento e morte de pequenas empresas. Questiona-se, no caso das pequenas empresas industriais, se os administradores conhecem e utilizam as informações que são relevantes acerca do seu negócio garantindo com isto uma gestão que leve a sobrevivência e crescimento.

Uma das formas de dar suporte a essas organizações é através da percepção e domínio de informações realmente úteis e relevantes a serem consideradas na análise e gerenciamento de suas atividades, pois se conhecendo as variáveis que influenciam o processo decisório tem-se a possibilidade de análises mais claras e fundamentadas no contexto competitivo dessas organizações.

A informação é um recurso estratégico para a gestão dos negócios. Segundo Vasconcelos e Cyrino (2000: 34) "A importância do conhecimento da informação e da tecnologia para a performance das empresas tem sido amplamente reconhecida por diversas correntes teóricas". Dessa forma, pode ser feita uma relação direta entre a vantagem competitiva e a informação: a vantagem competitiva é obtida através da prática de estratégias definidas e amparadas por um processo decisório que se baseia em informações obtidas em sistemas de informações, que, nesse caso, se estrutura de modo a permitir o gerenciamento das atividades da Cadeia de Valor. A informação é útil na elaboração e também na execução da estratégia organizacional, através do auxílio à tomada de decisão acerca das atividades da organização.

Neste contexto, McGee \& Prusak (1994:37) comentam que “(...) implícita ou explicitamente, a estratégia competitiva (cuidadosamente definida ou existente de maneira informal) é manifestada em processos organizacionais específicos que transformam vários recursos (capital, tecnologia, trabalho, matérias-primas, etc.) em produtos e serviços que fornecem valor aos clientes".

Nas empresas de menor porte normalmente este processo de definição e consecução de estratégias não ocorre de modo articulado. Assim, "a estratégia de pequenas empresas pode apenas 'acontecer' ou pode resultar de uma reflexão cuidadosa sobre a missão da empresa. Esta é obviamente preferível quando se monta uma pequena empresa de sucesso" (Longenecker et al, 1998:51). Disto infere-se que a estratégia está diretamente ligada ao resultado de uma empresa, com uma estratégia definida, torna-se mais claro o gerenciamento das atividades. É uma espécie de inter-relação: a estratégia irá auxiliar no 
gerenciamento das atividades, que, se bem executadas, irão auxiliar na definição e consecução da estratégia, e para isso são necessárias informações.

\section{A informação nas Pequenas Empresas}

Conforme afirmam Beraldi \& Escrivão Filho (2000: 48). “A grande motivação para que as pequenas empresas estejam buscando adquirir recursos da tecnologia da informação é a sua sobrevivência num mercado cada vez mais global e competitivo". As vantagens que podem ser obtidas com a tecnologia da informação são diversas e podem ser exploradas em grandes e pequenas organizações.

De acordo com Porter \& Millar (1985), a tecnologia da informação pode auxiliar no processo de implementação da estratégia e, com o uso dos sistemas de informação, as empresas podem mensurar suas atividades mais precisamente motivando seus gerentes a implementem estratégias de sucesso.

Com a diminuição considerável dos custos de computadores e programas e com o recente interesse das empresas de informática nos negócios de menor porte, já é possível às pequenas empresas o uso de recursos dos sistemas informatizados. Mas, mesmo assim, muitas empresas de pequeno porte ainda não se utilizam desses recursos que, segundo Beraldi \& Escrivão Filho (2000), estaria relacionado a dificuldade em obter financiamento, por falta de linhas de crédito adequadas ou por dificuldades em preencher as exigências necessárias.

Apesar dessas dificuldades o uso da TI por pequenas empresas é algo imprescindível, pois, como comenta Beuren (1998):

“(...) a informação funciona como um recurso essencial na definição da estratégia empresarial. Ademais, o conjunto de informações necessárias à elaboração da estratégia torna-se cada vez mais complexo em função da velocidade do movimento dos agentes de mercado. Atualmente, centenas de relações precisam ser consideradas para gerar uma informação competitiva, o que requer o gerenciamento sistemático e dinâmico da informação." (1998: 46).

A informação também é recurso na execução da estratégia:

A informação desempenha papéis importantes tanto na definição quanto na execução de uma estratégia. Isso se dá em relação ao aperfeiçoamento da definição de estratégias competitivas, à capacidade de executar estas estratégias ou à habilidade necessária para garantir que estratégias e execução permaneçam sincronizadas entre si e com o ambiente competitivo (Rezende \& Abreu, 2000:109).

Essas estratégias - genéricas, funcionais, corporativas, de mix de marketing, ou outro tipo, entre os inúmeros defendidos pela comunidade científica - devem ser fundamentadas e adequadas à realidade de cada empresa e meio, para que se possa efetivar, e, preferencialmente, garantir vantagens.

\section{A Cadeia de Valor e a Informação}

As atividades que compõe a Cadeia de Valor - atividades primárias (logística interna, operações, logística externa, marketing e vendas e serviços) e atividades de apoio (aquisição, desenvolvimento de tecnologia, gerência de recursos humanos e infra-estrutura da empresa) - necessitam de um efetivo gerenciamento para que possam adicionar valor aos produtos gerados e dar condições a empresa de buscar vantagens. Segundo Porter (1990), a vantagem competitiva não pode ser compreendida observando-se a empresa 
como um todo. Ela tem sua origem nas inúmeras atividades distintas que uma empresa executa no projeto, na produção, no marketing, na entrega e no suporte de seu produto.

O sucesso em uma organização depende do sucesso de suas atividades. O gerenciamento efetivo das atividades propiciará a agregação de valor aos produtos e/ou aos serviços. Agregar valor é fazer com que os investimentos realizados pela organização propiciem retorno (Porter, 1990), e uma das formas de fazê-lo é diferenciando-se em algum aspecto de alguma atividade de valor ou do gerenciamento dos elos entre elas. E para diferenciar-se, é necessário, inicialmente, conhecer as atividades de valor e administrá-las efetivamente.

A Cadeia de Valor apresenta-se como uma estrutura sobre a qual podem ser vislumbradas as atividades que merecem melhor gerenciamento, o que pode se dar através de informações geradas pelas e para essas atividades. Utilizando-se da estrutura base da Cadeia de Valor, é possível verificar se, em alguma das atividades, que compõem a Cadeia, a empresa possui algum diferencial que lhe propicie, ou auxilie na competitividade.

Por apresentar uma estrutura clara, inicialmente simples, a Cadeia de Valor parece se adaptar também aos negócios menores. As atividades do grupo inicial podem ser subdivididas de acordo com o ramo de cada negócio e seu porte, limitando, positivamente, as atividades a serem vislumbradas dentro do processo.

Prosseguindo, Porter expõe que "a definição de atividades de valor relevantes exige que atividades com economias e tecnologias distintas sejam isoladas. Funções gerais como fabricação ou marketing devem ser subdivididas em atividades" (1990: 41). Sobre estas é necessário definir as informações fundamentais, visto que "cada atividade de valor cria e usa informações de algum tipo" (Porter \& Millar, 1985:67).

Especificamente, quanto aos sistemas de informações - enquanto geradores das informações - e sua relação com as atividades da Cadeia de Valor, Porter (1990) comenta que os sistemas de informações são empregados em programação, controle, otimização, avaliação e em outras atividades. A tecnologia de sistemas de informações também tem um importante papel nos elos entre atividades de todos os tipos, porque a coordenação e a otimização exigem um fluxo de informações entre as atividades.

As atividades de valor precisam ser bem desempenhadas e gerenciadas para que a estratégia possa ser formulada de modo claro e efetivo. Do sucesso do gerenciamento das atividades depende o sucesso da organização. Saber a situação de cada atividade possibilita melhorar o seu desempenho no resultado global da organização.

\section{O Modelo Proposto}

A metodologia utilizada nesse estudo para o levantamento das informações fundamentais ao gerenciamento das atividades das pequenas empresas apresenta a seguinte seqüência:

a - a empresa deve ter as funções da Cadeia de Valor sub-divididas de acordo com as características específicas de cada setor/segmento/porte;

$\mathrm{b}$ - levantamento das informações julgadas relevantes a administração de cada uma das atividades na visão do administrador do negócio (que pode conter limitações, tendo em vista que os pequenos negócios muitas vezes são administradores por pessoas que não dominam todos os conhecimentos necessários a um efetivo gerenciamento);

c - diagnóstico e sistematização da percepção da empresa pelo seu administrador (através de um questionário semi-estruturado que trabalha com o modelo das 5 forças competitivas de Porter) e como a empresa administrada atua nesse ambiente;

d - sistematização dos dados e geração de uma lista das informações consideradas fundamentais na visão do administrador; 
e - submissão desses dados a análise de um grupo de especialistas detentores de conhecimento na área, que através da Técnica Delphi, no intuito de avaliar e gerar uma nova lista de informações consideradas críticas na perspectiva dos especialistas;

f - confrontação das listas geradas pelos empresários e especialistas de modo a promover um refinamento nas informações críticas ao gerenciamento da cadeia de valor.

A figura a seguir, dá uma idéia do método de chegar-se a lista final.
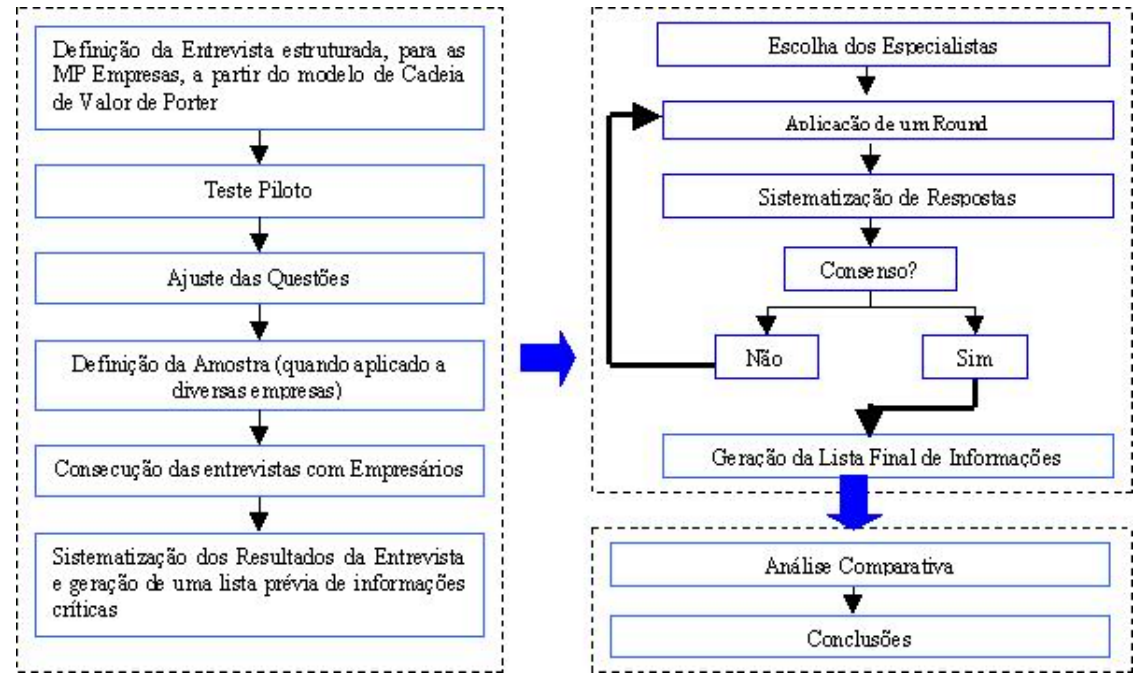

Figura 1 - Desenho do Modelo

\section{Aplicação do Modelo}

A aplicação do modelo ocorreu com uma amostra de pequenas empresas de beneficiamento, moagem, torrefação e fabricação de produtos alimentares de origem vegetal. Segundo o Cadastro Empresarial do Rio Grande do Sul (SEBRAE, 2000), há um universo de 390 empresas nesse ramo e dessas, 233 são micro e 116 são pequenas empresas, considerando o número de funcionários.

Em estudo anterior realizado no setor envolvendo médias e grandes indústrias, aponta-se que, devido à necessidade de se estar atento a algumas especificidades como, por exemplo, o desenvolvimento da matéria-prima junto ao produtor e o prazo limite que esta possui para ser processada, o processo decisório torna-se cada vez mais complexo, pela quantidade de elementos envolvidos e pela necessidade de tomar decisões de forma rápida (Luciano, Freitas \& Becker, 2000).

O desenvolvimento do modelo iniciou com a divisão dos grandes grupos de atividades em sub-atividades. No levantamento de dados foram realizadas visitas e entrevistas com os administradores utilizando como base um questionário semi-estruturado que dividiu a empresa em diversas atividades, tomando por base os grandes grupos de atividades da Cadeia de Valor de Porter. Esse questionário foi dividido em: 1) caracterização da empresa; 2) diagnóstico de como a empresa vê o ambiente no qual está inserida; 3) como a empresa se defende no ambiente no qual está inserida; 4) gerenciamento das Atividades da Cadeia de Valor.

Com este questionário pretendeu-se conhecer como os gestores percebem a empresa no atual ambiente competitivo, seu modo de ação e, especialmente, verificar quais as informações consideradas importantes no gerenciamento das atividades do negócio.

Seguindo a metodologia anteriormente detalhada, foi gerada uma lista final, que se apresenta como um modelo com a estrutura da Cadeia de Valor indicando quais são as informações fundamentais no gerenciamento das atividades da Cadeia das empresas em questão. Essas informações auxiliam no conhecimento e na melhor exploração e proveito das atividades, que podem ser o diferencial das empresas, que, se bem administradas, auxiliam diretamente na obtenção de vantagens competitivas. Desta forma, é possível 
elencar o grupo das informações mais importantes, ou seja, fundamentais ao gerenciamento das atividades, na visão de administradores de negócios e especialistas. As informações são as seguintes, por atividade dentro de cada função:

\begin{tabular}{|c|c|}
\hline \multicolumn{2}{|c|}{ LOGÍSTICA INTERNA ("grande atividade") } \\
\hline Recebimento de Matéria-Prima (sub-atividade) & Armazenagem de Matéria-prima \\
\hline $\begin{array}{l}\text { Informações: } \\
\text { - Qualidade laboratorial } \\
\text { - Evolução das despesas com a atividade } \\
\text { - Ganhos/perdas de produtividade }\end{array}$ & $\begin{array}{l}\text { Informações: } \\
\text { - Controle quantitativo de estoques } \\
\text { - Controle qualitativo de estoques } \\
\text { - Evolução perdas (\$) de qualidade } \\
\text { - Evolução Custos estocagem e reposição }\end{array}$ \\
\hline
\end{tabular}

\begin{tabular}{|l|l|}
\hline \multicolumn{2}{|c|}{ LOGÍSTICA EXTERNA } \\
\hline \multicolumn{1}{|c|}{ Armazenagem de Produtos Acabados } & \multicolumn{1}{c|}{ Distribuição dos Produtos Acabados } \\
\hline \multicolumn{1}{|c|}{ Informações: } & \multicolumn{1}{c|}{ Informações: } \\
- Evolução dos custos com armazenagem & - Avaliação roteiros (otimização) \\
- Perdas (\$) por problemas de validade & - Satisfação clientes (pesquisa) \\
& - Prazos médios de entrega x prazos concorrentes \\
& - Evolução custos de transporte \\
& - Perdas (\$) por problemas de transporte \\
\hline
\end{tabular}

\begin{tabular}{|l|l|l|}
\hline \multicolumn{2}{|c|}{ OPERAÇÕES } \\
\hline \multicolumn{1}{|c|}{ Produção } & \multicolumn{1}{|c|}{ Embalagem dos produtos } & Conserv. e manutenção Eqptos. \\
\hline \multicolumn{1}{|c|}{ Informações: } & \multicolumn{1}{|c|}{ Informações: } & \multicolumn{1}{c|}{ Informações: } \\
- Satisfação clientes & - Índice de problemas com & - Perdas (\$) por retrabalho \\
- Quantidade programada x & qualidade - evolução & - Custos de manutenção - evolução \\
produzida & - Perdas (\$) devido problemas & \\
- Evolução custos (\$) da empresa e & - Evolução custos & \\
concorrentes & & \\
- Produtividade & & \\
- Desperdícios e ociosidade & & \\
\hline
\end{tabular}

\begin{tabular}{|c|c|}
\hline \multicolumn{2}{|c|}{ LOGÍSTICA EXTERNA } \\
\hline Propaganda & Força de vendas \\
\hline $\begin{array}{l}\text { Informações: } \\
\text { - Reconhecimento da marca (pesquisa) } \\
\text { - Clientes conquistados (pesquisa/evolução) } \\
\text { - Evolução das vendas (\$) }\end{array}$ & $\begin{array}{l}\text { Informações: } \\
\text { - Vendas (\$) - orçado x executado } \\
\text { - Crescimento de vendas por cliente } \\
\text { - Crescimento parcela de mercado }\end{array}$ \\
\hline $\begin{array}{l}\text { Fixação de preços } \\
\end{array}$ & Canais de distribuição \\
\hline $\begin{array}{l}\text { Informações: } \\
\text { - Evolução vendas (quantidade) } \\
\text { - Evolução vendas (\$) } \\
\text { - Satisfação consumidor (pesquisa) }\end{array}$ & $\begin{array}{l}\text { Informações: } \\
\text { - Evolução dos custos com a distribuição } \\
\text { - Evolução do faturamento por canal } \\
\text { - Valor (\$) da inadimplência }\end{array}$ \\
\hline
\end{tabular}

\begin{tabular}{|l|}
\hline \multicolumn{1}{|c|}{ SERVIÇOS } \\
\hline \multicolumn{1}{|c|}{ Serviços agregados aos produtos } \\
\hline - Satisfação clientes (pesquisa) \\
- \% Clientes estáveis (fiéis) \\
$-\%$ Clientes atendidos, mas insatisfeitos (pesquisa) \\
\hline
\end{tabular}

\begin{tabular}{|l|l|}
\hline \multicolumn{2}{|c|}{ INFRA-ESTRUTURA DE APOIO } \\
\hline \multicolumn{1}{|c|}{ Gerência Geral } & \multicolumn{1}{c|}{ Gerência Financeira } \\
\hline \multicolumn{1}{|c|}{ Informações: } & \multicolumn{1}{c|}{ Informações: } \\
- Metas atingidas & - Satisfação sócios/acionistas \\
- Imagem da empresa (pesquisa) & - Situação do fluxo de Caixa \\
- Satisfação clientes internos e externos (pesquisa) & - Liquidez da empresa \\
- Crescimento real da empresa & - Lucratividade \\
- Resultado final (lucro) - evolução & \multicolumn{1}{|c|}{ Contabilidade } \\
\hline \multicolumn{1}{|c|}{ Qualidade } \\
\hline - Satisfação do usuário com as inf. geradas (pesquisa) & - Adequação aos padrões legais \\
\hline
\end{tabular}




\begin{tabular}{|c|c|c|}
\hline & & $\begin{array}{l}\text { - Índice de devoluções } \\
\text { - Perdas/ganhos (\$) com qualidade (ou falta de) }\end{array}$ \\
\hline \multicolumn{3}{|c|}{ GERÊNCIA DE RECURSOS HUMANOS } \\
\hline Recrutamento & Treinamento & Controle da Satisfação \\
\hline $\begin{array}{c}\text { Informações: } \\
\text { - Rendimento (produtividade) }\end{array}$ & $\begin{array}{l}\text { Informações: } \\
\text { - Incremento na produtividade } \\
\text { - Evolução qualidade / defeitos / } \\
\text { falhas } \\
\text { - Retorno sobre o investimento (\$) }\end{array}$ & $\begin{array}{l}\text { Informações: } \\
\text { - Absenteísmo } \\
\text { - Rotação de pessoal }\end{array}$ \\
\hline
\end{tabular}

\begin{tabular}{|l|l|}
\hline \multicolumn{2}{|c|}{ DESENVOLVIMENTO DE TECNOLOGIAS } \\
\hline \multicolumn{1}{|c|}{ Novas Tecnologias em equipamentos/processos } & \multicolumn{1}{c|}{ Novos Produtos } \\
\hline \multicolumn{1}{|c|}{ Informações: } & \multicolumn{1}{c|}{ Informações: } \\
- Produtividade \\
$\begin{array}{l}\text { Custos de manutenção } \\
\text { Retorno sobre investimentos }\end{array}$ & - Atendimento necessidades dos clientes (pesquisa) \\
\hline
\end{tabular}

\begin{tabular}{|lc|}
\hline & AQUISIÇÃO \\
\hline & Função de aquisição \\
\hline & Informações: \\
- Qualidade aquisições & \\
- Evolução reflexo nos preços de venda & \\
\hline
\end{tabular}

A lista gerada apresenta $60 \%$ das informações inicialmente elencadas pelos administradores de negócios, que, então, receberam a concordância dos especialistas. A maioria das atividades que os empresários elencaram como fundamentais, e que acredita-se que sejam as utilizadas no gerenciamento das organizações, são fundamentais na visão também do especialista. $\mathrm{O}$ empresário deve dar, assim, maior ênfase às informações tidas pelos especialistas como fundamentais, visando aproveitar àquelas que são realmente úteis para a avaliação e administração das atividades.

$\mathrm{O}$ uso das informações fundamentais propicia certa garantia de que a análise e o gerenciamento estarão sendo feitos com menores chances de erros. Desta forma, o grupo final elencado como fundamental poderá ser a base de consulta para os administradores das empresas na sua prática diária de tomada de decisões.

É relevante destacar o fato de que a maioria das informações indicadas, no caso específico, é de caráter retrospectivo, trazendo algumas restrições no que se refere à implementação de uma estratégia que garanta vantagens competitivas. As informações indicadas permitem o gerenciamento das atividades, pensado-se no momento atual da empresa. No entanto, com alguns indicadores prospectivos o auxílio à obtenção e garantia da competitividade torna-se mais exeqüível. Tanto empresários quanto especialistas limitaram-se a uma perspectiva mais tática do que estratégica, com um posicionamento um tanto limitado no que concerne às necessidades informacionais para a obtenção de vantagens competitivas. Sente-se a falta de uma certa "ousadia" nas opiniões expressas. As informações indicadas podem auxiliar na consecução das estratégias, mas somente serão suficientes se acompanhadas de um novo conjunto com perspectivas de futuro.

\section{Conclusão}

O presente estudo pretende dar uma pequena contribuição, no sentido de sugerir um modelo que demonstre quais as informações consideradas fundamentais na gestão das atividades da Cadeia de Valor de um grupo de empresas industriais de pequeno porte de beneficiamento, moagem, torrefação e fabricação de produtos alimentares de origem vegetal, na ótica de empresários e um conjunto de especialistas. Todas as atividades desses negócios merecem atenção, pois, ora individualmente ora em conjunto, são responsáveis, direta ou indiretamente, pelo sucesso ou insucesso da organização. Pelas atividades e seu 
efetivo gerenciamento, é possível buscar e praticar formas diferentes no mercado (seja em custos, produtos ou serviços, processos), para cativar clientes, ser competitivo e buscar vantagens.

No gerenciamento dessas atividades, as informações são recursos preciosos. Informações confiáveis respaldam decisões. A informação sozinha não garante o sucesso, mas se usada, efetivamente, por profissionais capacitados com staff de apoio, reduz as chances de insucesso da empresa. A informação traduz-se, hoje, como um recurso em todos os níveis de uma empresa, auxiliando a gestão. Soluções elaboradas a partir de necessidades específicas, como o modelo apresenta, possuem maiores chances de atender as expectativas e espelhar a realidade.

\section{Referências Bibliográficas}

BERALDI, Lirce Castanhera; ESCRIVÃO FILHO, Edmundo. Impacto da tecnologia de informação na gestão de pequenas empresas. Revista Ciência da Informação - IBICT, Brasília, v. 29, n. 1, pg. 46-50, jan./abr. 2000. Disponível em: http://www.ibict.br/cionline/290100/29010005.pdf Acesso em 10/09/2001.

BEUREN, Ilse Maria. Gerenciamento da informação: um recurso estratégico no processo de gestão empresarial. São Paulo: Atlas, 1998.

CÂNDIDO, Genisaldo Ataíde; ABREU, Aline França de. Aglomerados industriais de pequenas e médias empresas como mecanismo para promoção de desenvolvimento regional. Revista Eletrônica de Administraçãa. UFRGS. Edição 18, n. 6, vol. 6. http://read.adm.ufrgs.br/read18/artigo/artigo4.htm Acesso em 15/09/2001.

COHEN, David. Como se faz gente que faz? Exame, São Paulo, ed. 721, ano 34, n. 17, p. 158-167, 23 ago. 2000.

FREITAS, Henrique (et al). Informação e decisão: sistemas de apoio e seu impacto. Porto Alegre: Ortiz, 1997.

LONGENECKER, J. G.; MOORE, C. W.; PRETTY, J. W. Administração de Pequenas

Empresas. São Paulo: Makron Books, 1998.

LUCIANO, Edimara Mezzomo; FREITAS, Henrique M. Rodrigues de; BECKER, João Luiz. Perfil decisório da indústria alimentar gaúcha. Revista Eletrônica de Administração. Ed. 16, n. 4, vol. 6, nov. 2000. Disponível em http://read.adm.ufrgs.br/read16/artigo/artigo2.htm Acesso em 20/01/2001.

MCGEE, James; PRUSAK, Laurence. Gerenciamento estratégico da informação. Rio de Janeiro: Campus, 1994.

PORTER, Michael E. Estratégia Competitiva. 7. ed. Rio de Janeiro: Campus, 1986.

PORTER, Michael E. Vantagem Competitiva. Rio de Janeiro: Campus, 1990.

PORTER, Michael E; MILLAR, Victor. Como a informação lhe proporciona vantagem competitiva. 1985. In: McGOWAN, William (org). Revolução em tempo real. Rio de Janeiro: Campus, 1997.

REZENDE, Denis Alcides; ABREU, Aline França de. Tecnologia da informação aplicada a sistemas de informações empresariais. São Paulo: Atlas, 2000.

SEBRAE - Serviço Brasileiro de Apoio a Micro e Pequena Empresa. Fatores condicionantes e taxa de mortalidade de empresas. Brasília: Ed. Sebrae, 1999.

Disponível em http://200.252.248.103/sites/doc pdf/estudos e pesquisas/arquivos/mortalempresas.pdf Acesso em 05/06/2001.

SEBRAE. Cadastro Empresarial do Rio Grande do Sul de 2000. 2000. CD-ROM. Produzido por Microservice Tecnologia Digital S/A.

VASCONCELOS, Flávio C.; CYRINO, Álvaro B. Vantagem competitiva: os modelos teóricos atuais e a convergência entre estratégia e teoria organizacional. Revista de

Administração de Empresas, São Paulo, v. 40, n. 4, p. 20-37, out./dez. 2000. 